\title{
Qi-Long-Tian formula extract alleviates symptoms of acute high-altitude diseases via suppressing the inflammation responses in rat
}

\author{
Xing Fu' ${ }^{1}$, Chunyan Yang ${ }^{2}$, Bing Chen ${ }^{3}$, Kexing Zeng ${ }^{4}$, Siyuan Chen ${ }^{4}$ and $\mathrm{Yi} \mathrm{Fu}^{5^{*}}$
}

\begin{abstract}
Background: Chinese Yunnan Province, located in the Yunnan-Guizhou Plateau, is a famous tourist paradise where acute high-altitude illness common occurs among lowland people visitors due to non-acclimatization to the acute hypobaric hypoxia (AHH) conditions. Traditional Chinese medicine, such as Qi-Long-Tian (QLT) formula, has shown effectiveness and safety in the treatment of acute high-altitude diseases. The aim of this study was to clarify the therapeutic mechanisms of this traditional formula using a rat model in a simulated plateau environment.
\end{abstract}

Methods: Following testing, lung tissue samples were evaluated by hematoxylin-eosin staining and for biochemical characteristics. mRNA-Seq was used to compare differentially expressed genes in control rats, and in rats exposed to $\mathrm{AHH}$ and $\mathrm{AHH}$ with QLT treatment.

Results: Inflammation-related effectors induced following QLT treatment for AHH included MMP9 and TIMP1, and involved several phosphorylation signaling pathways implicated in AHH pathogenesis such as PI3K/AKT and MAPK signaling.

Conclusion: This study provides insights into the major signaling pathways induced by $\mathrm{AHH}$ and in the protective mechanisms involved in QLT formula activity.

Keywords: Acute hypobaric hypoxia, Qi-Long-Tian, mRNA-seq, Acute high-altitude diseases, Inflammation

\section{Background}

The northern part of Yunnan Province, China is located on a plateau with an average altitude of over $2000 \mathrm{~m}$. With increasing altitude, the atmospheric pressure decreases exponentially, resulting in a progressive reduction in the ambient partial pressure of oxygen $\left(\mathrm{PO}_{2}\right)$, termed hypobaric hypoxia. When the altitude increases by $100 \mathrm{~m}$, the atmospheric pressure usually drops by $5 \mathrm{mmHg}$, and the

\footnotetext{
*Correspondence: fuyi7510@sina.com

${ }^{5}$ The Third Affiliated Hospital of Yunnan University of Chinese Medicine, Kunming Municipal Hospital of Traditional Chinese Medicine, No. 2628 Xiangyuan Street, Chenggong District, Kunming 650500, Yunnan, China Full list of author information is available at the end of the article
}

$\mathrm{PO}_{2}$ drops by $1 \mathrm{mmHg}$. Millions of individuals, including the military, mountain rescuers, and mountaineers, currently work or live at high altitudes, and are exposed to the risk of mountain sickness. Physiological studies have shown that the altitude suitable for human survival is between 500 and $2000 \mathrm{~m}$ [1]. Individuals experience difficulty breathing and altitude symptoms appear above $2000 \mathrm{~m}$, while saliva secretion begins to decrease at $3000 \mathrm{~m}$. Most individuals experience a state of incapacitation at $6000 \mathrm{~m}$ and loss of consciousness and hallucinations occur at $7000 \mathrm{~m} \mathrm{[2]}$.

When lowland people visit plateaus, a syndrome caused by poor adaptability to the acute hypobaric hypoxia $(\mathrm{AHH})$ environment called acute mountain original author(s) and the source, provide a link to the Creative Commons licence, and indicate if changes were made. The images or other third party material in this article are included in the article's Creative Commons licence, unless indicated otherwise in a credit line to the material. If material is not included in the article's Creative Commons licence and your intended use is not permitted by statutory regulation or exceeds the permitted use, you will need to obtain permission directly from the copyright holder. To view a copy of this licence, visit http://creativecommons.org/licenses/by/4.0/. The Creative Commons Public Domain Dedication waiver (http://creativeco mmons.org/publicdomain/zero/1.0/) applies to the data made available in this article, unless otherwise stated in a credit line to the data. 
sickness (AMS) may occur [3]. The principal symptoms of AMS include headache, nausea, vomiting, fatigue, dizziness, and sleep disturbance [4]. Although AMS has been recognized over the past two centuries, little is known about the fundamental causes of these symptoms [5]. Yunnan Province is a tourist paradise, where the number of tourists exceeded 600 million in 2020 . It is foreseeable that AMS would seriously threaten the health of travelers visiting the plateau.

A series of physiological responses occur to adapt to AHH conditions. In some cases, these maladaptive responses may predispose affected individuals to various forms of high-altitude illness. The lungs are the first organ to sense changes in barometric pressure and the $\mathrm{PO}_{2}$ in the environment and are closely associated with the body's maladaptive responses to $\mathrm{AHH}$ [6], which result in significant injuries caused by hypobaric hypoxia, such as high-altitude pulmonary edema (HAPE).

HAPE is noncardiogenic pulmonary edema that may occur in unacclimatized persons within 2 to 4 days of ascent to altitudes above $2500 \mathrm{~m}$ [7]. Clinical findings include tachycardia, tachypnea, low-grade fever, hypoxemia, and unilateral or bilateral inspiratory crackles [8]. Risk factors for HAPE include a prior history of the disorder, male sex, rapid ascent, higher altitudes, pre-existing respiratory infection, and intense exercise [9-11]. Susceptible individuals develop exaggerated hypoxic pulmonary vasoconstriction and may experience a large rise in pulmonary artery pressure when exposed to hypoxia. As these changes are distributed unevenly within the pulmonary vascular bed, regional over perfusion of capillaries occurs, leading to a 'stress failure' of the blood-gas barrier, increased permeability, and pulmonary edema [12]. This noninflammatory process may be accelerated by impaired alveolar fluid clearance [13].

Traditional Chinese medicine (TCM), which has long been practiced in the clinic in Asian countries, is an important choice for preventing and treating disease. Formulae based on TCM theories are reported to be effective in treating hypoxia pulmonary hypertension which is a symptom of HAPE at the early stage. The QiLong-Tian (QLT) formula is composed of three herbs (Rhodiola, Panax notoginseng, and dried pheretima at a ratio of 10:5:2) [14]. As a component of QLT, Rhodiola has been used in the clinic as a lung protecting agent and can improve cardiopulmonary function, alleviate pulmonary hypertension, and reduce the probability of the occurrence of HAPE $[15,16]$. A previous study has shown that this formula reduced endothelin-1, vascular endothelial growth factor, interleukin (IL)-1 $\beta$, and tumor necrosis factor (TNF)- $\alpha$ levels, and upregulated nitric oxide synthase (NOS) expression in experimental rats with hypoxia pulmonary hypertension $[17,18]$, but the underlying mechanisms are still unclear.

In the present study, we investigated the mRNA profile of lung tissues from rat models exposed to conditions of normoxia, $\mathrm{AHH}$, or to $\mathrm{AHH}$ with Qi-Long-Tian treatment. The results showed that several inflammationrelated mediators were significantly induced under $\mathrm{AHH}$ conditions, which also responded to QLT treatment. We deduce that these molecule candidates may mediate the action of QLT in vivo. This study provides deeper insight into the mechanisms of action of QLT in the treatment of AHH-related diseases.

\section{Methods}

\section{Preparation of the extract of QLT formula}

The composition of the QLT formula includes Rhodiola sachalinensis, Panax notoginseng, and dried Pheretima asiatica at a ratio of 10:5:2. The raw medicinal materials were obtained and prepared by the Kunming Municipal Hospital of TCMs. The materials were diluted 10 times in distilled water $(\mathrm{w} / \mathrm{v})$ and boiled twice for $1 \mathrm{~h}$ each time under continuous stirring. After filtration, the residue was boiled with 8 volumes of purified water for another $2 \mathrm{~h}$ and filtered. Thereafter, the combined filtrates were subjected to centrifugation at $1500 \times g$ and the supernatants were concentrated under vacuum to a final concentration of $2 \mathrm{~g} / \mathrm{mL}$ and stored at $-80^{\circ} \mathrm{C}$ until use.

\section{Animals}

Specific pathogen-free male Wistar rats $(230 \pm 20$ g; 6-weeks-old) were obtained from the Laboratory Animal Center of Kunming Medical University. All rats were maintained in an environment at $23 \pm 2{ }^{\circ} \mathrm{C}$ with $50 \pm 5 \%$ humidity and a $12 \mathrm{~h}$ light-dark cycle. Water and food were provided to animals ad libitum. The experiments started after a week of adaptation. The experimental protocol was approved by the Experimental Animal Care and Ethics Committees of Kunming Municipal Hospital of Traditional Chinese Medicine (Approval No.: R-082018112).

\section{Rat model preparation and administration}

The 130 rats were randomized and divided into the blank (I), AHH (II), and AHH with QLT treatment (III) groups. Animals of the blank group $(n=10)$ remained at sea-level atmospheric pressure within the same laboratory conditions. Animals of groups II and III (60 rats for each) were exposed to $\mathrm{AHH}$ for a total of 24,48 , or $72 \mathrm{~h}$ (20 rats for each duration) in a decompression chamber (Feng Lei, Guizhou, China) at a simulated altitude of $6000 \mathrm{~m}$. The animals of the hypobaric hypoxia group III received $8 \mathrm{~g} /$ $\mathrm{kg}$ (20 rats for each duration) QLT by gavage with once a day for the duration of the experiment. The rats of group 
II received the same volume of saline intragastrically. The airflow of the hypobaric hypoxia decompression chamber was maintained $10 \mathrm{~L} / \mathrm{min}$ during exposure to prevent the accumulation of exhaled gases.

\section{Serum samples}

The rats were euthanized with $\mathrm{CO}_{2}$ asphyxia for $5 \mathrm{~min}$. Blood samples ( $4 \mathrm{~mL}$ from each individual rat) were collected from the carotid artery into 5\% volume heparin tubes (Venoject II, Terumo, Tokyo, Japan) and immediately centrifuged at $3000 \times g$ for $10 \mathrm{~min}$ at $4{ }^{\circ} \mathrm{C}$. The serum was separated and stored at $-80^{\circ} \mathrm{C}$ until analysis.

\section{Hematoxylin-eosin (HE) staining}

Paraffin tissue sections were dehydrated under standard protocols, including clearing in xylene (3 times), and dehydration in anhydrous alcohol (95\% alcohol, $80 \%$ alcohol, 75\% alcohol), and distilled water, successively. Paraffin tissue sections were stained with hematoxylin for $5 \mathrm{~min}$, rinsed using tap water, and blotted dry. Next, they were differentiated using ethanolic acid for $30 \mathrm{~s}$, soaked in warm water at $50{ }^{\circ} \mathrm{C}$ for $5 \mathrm{~min}$, and drained. Slides were incubated with eosin stain for $2 \mathrm{~min}$ and followed by routine dehydration, including $95 \%$ alcohol, anhydrous alcohol, xylene carbolic acid, xylene, in order. Finally, slides were sealed with a neutral resin.

\section{Immunohistochemistry}

The tissue specimens were embedded in paraffin and sectioned continuously. The sections were placed in a $65{ }^{\circ} \mathrm{C}$ oven overnight, then dewaxing and hydration were carried out. Endogenous peroxidase was blocked by $3 \%$ $\mathrm{H}_{2} \mathrm{O}_{2}$. The sections were placed in citrate buffer solution and a microwave heating method was used to repair the antigen. Next, the sections were blocked with $5 \%$ normal sheep serum at room temperature (RT) for $20 \mathrm{~min}$. Primary antibody was added at $4{ }^{\circ} \mathrm{C}$ overnight. A biotinconjugated secondary antibody (1:200) was added and incubated at $37^{\circ} \mathrm{C}$ for $30 \mathrm{~min}$. HRP-labeled streptavidin solution was added and incubated at $37^{\circ} \mathrm{C}$ for $30 \mathrm{~min}$. After DAB staining, the sections were dyed with hematoxylin at RT for $2 \mathrm{~min}$, then dehydrated and sealed with neutral resin. The following antibodies were used: CD3 (1:150, Abcam, ab16669), CD68 (1:50, Abcam, ab955), HIF-1 $\alpha$ (1:1000, Abcam, ab8366), VEGF (1:100, Thermo, M808), CGB (1:600, Abcam, ab202972), EPO (1:500, Abcam, ab273070).

\section{RNA extraction}

RNA extraction was carried out as previously described [19]. In brief, 50-100 mg tissue was placed in a $1.5 \mathrm{~mL}$ EP tube (RNase free), and $1 \mathrm{~mL}$ Trizol was added to homogenize tissues. The tissue was allowed sit for $5 \mathrm{~min}$ at RT. Chloroform $(0.2 \mathrm{~mL})$ was added into an EP tube and mixed vigorously for $30 \mathrm{~s}$, and then allowed to sit for another $5 \mathrm{~min}$ at RT. Next, the lysate was centrifuged for $10 \mathrm{~min} 4{ }^{\circ} \mathrm{C}$ at $12,000 \times g$, and the liquid in the centrifuge tube produced different layers after centrifugation. The supernatant was carefully pipetted to a new $1.5 \mathrm{~mL}$ EP with a small pipette. Isopropanol $(0.5 \mathrm{~mL})$ was added and the mixture vigorously for $30 \mathrm{~s}$, and allowed to sit for another $10 \mathrm{~min}$ at RT. Then, lysate was centrifuged again for $10 \mathrm{~min} 4{ }^{\circ} \mathrm{C} 12,000 \times g$, leaving a precipitate at the bottom of the tube. Next, $75 \%$ ethanol $(1 \mathrm{~mL}$, precooled at $4{ }^{\circ} \mathrm{C}$ ) was added and the precipitate was suspended again. After that, the EP was centrifuged for $15 \min 4{ }^{\circ} \mathrm{C}$ at $12,000 \times g$, and the supernatant was discarded. An appropriate amount of diethylpyrocarbonate (DEPC) $\mathrm{H}_{2} \mathrm{O}$ (and incubated at $65{ }^{\circ} \mathrm{C}$ to promote dissolution) was added based on the size of the precipitation when the EP tube was air drying. The tube was oscillated to dissolve the precipitate. The concentration of total RNA was measured by Nanodrop.

\section{Total antioxidant capacity (TAOC) assay}

TAOC of the sera was determined colorimetrically at $570 \mathrm{~nm}$ according to the instructions of a commercial TAOC assay kit (Abcam).

\section{ELISA}

The levels of IL- $1 \beta$ in the blood serum were determined using a commercial ELISA kit (Abcam) according to the manufacturers' protocol.

\section{mRNA sequencing}

A total amount of $1 \mu \mathrm{g}$ RNA per sample was used as input material for the RNA sample preparations. Sequencing libraries were generated using NEBNext ${ }^{\circledR}$ Ultra $^{\mathrm{TM}}$ RNA Library Prep Kit for Illumina ${ }^{\circledR}$ (NEB, USA) following the manufacturer's recommendations and index codes were added to attribute sequences to each sample.

The clustering of the index-coded samples was performed on a cBot Cluster Generation System using TruSeq PE Cluster Kit v3-cBot-HS (Illumina) according to the manufacturer's instructions. After cluster generation, the library preparations were sequenced on an Illumina Novaseq platform and $150 \mathrm{bp}$ paired-end reads were generated.

\section{Sequencing data analysis}

Raw data (raw reads) of fastq format were first processed using in-house perl scripts. In this step, clean data (clean reads) were obtained by removing reads containing adapter sequences, reads containing ploy- $\mathrm{N}$ regions, and low-quality reads from raw data. At the same time, Q20, Q30, and GC content of the clean data were calculated. 
All the downstream analyses were based on clean data with high quality.

The reference genome and gene model annotation files were downloaded from the genome website directly. Index of the reference genome was built using Hisat2 v2.0.5 and paired-end clean reads were aligned to the reference genome using Hisat2 v2.0.5.

Differential expression analysis of the two conditions (two biological replicates per condition) was performed using the DESeq2 R package (1.16.1). DESeq2 provides statistical routines for determining differential expression in digital gene expression data using a model based on the negative binomial distribution. The resulting P-values were adjusted using the Benjamin and Hochberg's approach to control the false discovery rate. Genes with an adjusted P-value $<0.05$ defined as DESeq2, were assigned as differentially expressed.

\section{GO and KEGG enrichment analysis of differentially expressed genes}

Gene Ontology (GO) enrichment analysis of differentially expressed genes was implemented by the cluster profile $\mathrm{R}$ package, in which gene length bias was corrected. GO terms with a corrected $\mathrm{P}$-value $<0.05$ were considered significantly enriched by differentially expressed genes.

Kyoto Encyclopedia of Genes and Genomes (KEGG) is a database resource for understanding high-level functions and utilities of biological systems, such as the cell, the organism, and the ecosystem, using molecular-level information, especially using large-scale molecular datasets generated by genome sequencing and other highthroughput experimental technologies (http://www. genome.jp/kegg/). We used a cluster profile R package to test the statistical enrichment of differential expression genes in the KEGG pathways.

\section{Quantitative polymerase chain reaction}

Total RNA was reverse transcribed to cDNA using a Reverse Transcription Kit (Takara, Dalian, China). Realtime PCR analyses (qPCR) were performed with SYBR Green (Takara, Dalian, China) and the following amplification parameters were used: $95{ }^{\circ} \mathrm{C}$ for $1 \mathrm{~min}, 95{ }^{\circ} \mathrm{C}$ for $10 \mathrm{~s}$, and $60^{\circ} \mathrm{C}$ for $60 \mathrm{~s}$ (40 cycles). The amplification mixture included $50 \mathrm{ng} \mathrm{cDNA}, 2 \mathrm{mM} \mathrm{Mg}{ }^{2+}, 100 \mathrm{pmol}$ of each primer, $200 \mathrm{mM}$ dNTP, and $2.5 \mu \mathrm{g}$ Tag DNA polymerase. Results were normalized to the expression of glyceraldehyde-3-phosphate dehydrogenase (GAPDH) and the relative expression of genes was calculated using the $2^{-\Delta \Delta \mathrm{Ct}}$ method. The primers used are listed in Table 1. The ABI 7500 fast real-time PCR system (ABI, USA) was employed for the PCR analyses.
Table 1 The sequence of the primers used for qRT-PCR in this research

\begin{tabular}{ll}
\hline Gene & Primers sequence \\
\hline HIF-1a & Forward: 5'-CATCGAGCCACTCTTTGACC-3' \\
VEGF & Reverse: 5'-GCCATTAGGTAGGTGTAGCTGG-3' \\
& Forward: 5'-CCTGGCAAAGAGAAGACACAGTG-3' \\
CGB & Reverse: 5'-GAAGGGTAAGCCACTCACACAC-3' \\
& Forward: 5'-CATTCTTCCAGACCAGGG-3' \\
EPO & Reverse: 5'-CATCTTTGGTGTGCCTGAG-3' \\
& Forward: 5'-CTCTTCCGGGTCTACTCCAAC-3' \\
MMP8 & Reverse: 5'-GGTTCTCTCAGTCTCTCCTGCTC-3' \\
& Forward: 5'-CAATGGAATCCTTGCCCATG-3' \\
MMP9 & Reverse: 5'-GTGCTGGGTTCTCTGTAAGC-3' \\
& Forward: 5'-CCCTTGAACTAAGGCTCCTC-3' \\
IL-17b & Reverse: 5'-GACAAGCTGATTGGTTCGAG-3' \\
& Forward: 5'-CTGAGGAACAGCTCTGAGCC-3' \\
TIMP1 & Reverse: 5'-CTCACCATGCTACGGTCCTC-3' \\
& Forward: 5'-GAACCGCAGCGAGGAGTTC-3' \\
CCI2 & Reverse: 5'-CAAGCAATGACTGTCACTCTCC-3' \\
& Forward: 5'-GCAGCAGGTGTCCCAAAGAAG-3' \\
S100a8 & Reverse: 5'-GTGGAAAAGAGAGTGGATGC-3' \\
CCI7 & Forward: 5'-GAAGACAGATGCCTGAACAG-3' \\
& Reverse: 5'-GTGACATGAGGTCTCCAGG-3' \\
& Forward: 5'-GAGAATACTGCAGACCAGGTC-3' \\
& Reverse: 5'-GACTGTCCACCACCAACTC-3' \\
& Forward: 5'-GGAGCTGCAAAGACCAAAG-3' \\
& Reverse: 5'-CAGTCCCGTCATCTACAAAC-3' \\
& Forward: 5'-GGCAACTGAACTGGAGAAGG-3' \\
& Reverse: 5'-CCCTTATCACCAACACAAGG-3' \\
& Forward: 5'-CATCCTGACACCCTGAACAAG-3' \\
& Reverse: 5'-GTGGGTTGTTCTCATGCAG-3' \\
\hline &
\end{tabular}

\section{Western blot analysis}

The tissues were fully lysed and sonicated, and the total protein was extracted. Protein levels were quantified using a BCA Protein Assay Kit. Polyacrylamide gel electrophoresis was performed with $10 \mu \mathrm{g}$ protein from each sample. Next, proteins on the gel were transferred onto a polyvinylidene fluoride (PVDF) membrane by semi-dry transfer, and the PVDF membrane was sealed with 5\% skim milk powder for $2 \mathrm{~h}$ at RT. Thereafter, the membrane was probed with the primary antibody at 1:1000 dilution with phosphate buffer saline (PBS) against matrix metalloproteinase 8 (MMP8), MMP9, IL-17 $\beta$, tissue inhibitor of metalloproteinase 1 (TIMP1), Ccl2, Ccl7, TNF ligand superfamily member 9 (TNFSF9), and Fas apoptotic inhibitory molecule (FAIM), S100a8, S100a9 at $4{ }^{\circ} \mathrm{C}$ overnight. All primary antibodies were provided by Cell Signaling. The PVDF membrane was then washed 
three times with PBS-Tween for $8 \mathrm{~min}$ each. The secondary antibody was diluted with PBS and was incubated for $1 \mathrm{~h}$ at RT, and the PVDF membrane was washed again. $\beta$-actin was used as a loading control. Proteins were visualized using an enhanced chemiluminescence solution. The following antibodies were used: MMP8 (Abcam, ab81286), MMP9 (CST, 13667T), IL-17 $\beta$ (Abcam, ab125029), TIMP1 (CST, 8946S), Ccl2 (Abcam, ab186421), Ccl7 (Abcam, ab182793), TNFSF9 (Abcam, ab68185), FAIM (Abcam, ab154570), S100a8 (CST, 47310T), and S100a9 (CST, 73425S).

\section{Statistical analysis}

Statistical analysis was performed using PASW Statistics for Windows, Version 18.0 (SPSS Inc., Chicago, IL, USA) and GraphPad Prism Software (GraphPad Software, Inc., San Diego, CA, USA). Comparisons between the three groups were analyzed with one-way Analysis of Variance (ANOVA), and the multiple comparisons were analyzed with Tukey. A P-value $<0.05$ was considered to indicate significant differences. Each experiment was performed at least three times, and the results were expressed as the mean \pm standard deviation (SD).

\section{Results}

QLT1 alleviated lung injury and immune cell infiltration in a rat $\mathrm{AHH}$ model

We used hematoxylin-eosin staining to observe the damage to the lung tissue of rats under $\mathrm{AHH}$ conditions. The lung tissue of the blank group showed no obvious pathological changes, and the alveoli were regular and intact. There was no obvious edema of the alveolar interval nor immune cell infiltration. However, the alveolar septa thickened under $\mathrm{AHH}$ for $24 \mathrm{~h}$ and presented immune cell infiltration and capillary congestion. After $48 \mathrm{~h}$ the alveolar septa of the rats in AHH were broken; the lung tissue structure was destroyed at $72 \mathrm{~h}$, in addition changes in the alveolar septa, and the capillaries were dilated and a presented a large number of infiltrated inflammatory cells. QLT treatment significantly alleviated the structural changes at $72 \mathrm{~h}$ under AHH (Fig. 1a).

In lung tissues under AHH conditions, alveoli walls presented immune cell infiltrated. IHC staining of CD3 and CD68 were employed to assess $\mathrm{T}$ cell and macrophage cell infiltration, and to compare the level of inflammation between the AHH and QLT $+\mathrm{AHH}$ groups at the 72-h time point. $\mathrm{T}$ cells and macrophage cells were significantly infiltrated in the alveoli walls. While the QLT treatment significantly alleviated the degree of immune cell infiltration (Fig. 1b).

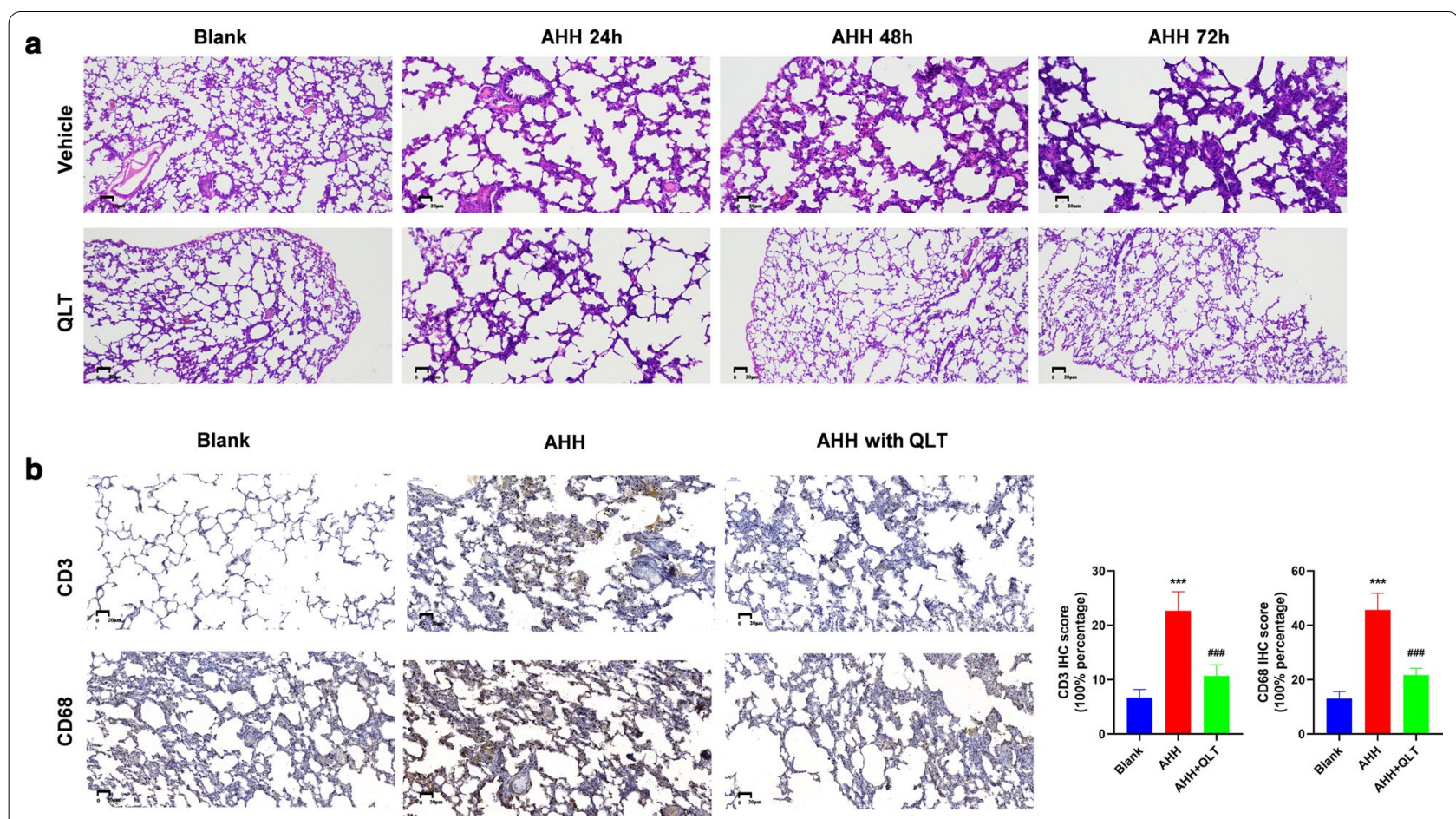

Fig. 1 Phenotype of rat lung after the rats were exposed to AHH or AHH and QLT treatment. a HE staining for pathological changes of rat lung of each group. $\mathbf{b} \| \mathrm{HC}$ staining of CD3 and CD68 of the rat lung of each groups after a 72-h exposure. ${ }^{* *} \mathrm{P}<0.001,{ }^{*}$ blank vs. AHH, ${ }^{*} A H H$ vs. QLT + AHH 


\section{QLT1 alleviated TAOC and hypoxia induces inflammation in rat $\mathrm{AHH}$ model}

TAOC reflects the capacity of the body's overall defense against $\mathrm{AHH}$-induced oxidative damage. TAOC significantly decreased at $48 \mathrm{~h}$ post- $\mathrm{AHH}$, and in the $\mathrm{AHH}$ group was one-third that of the blank group $(\mathrm{P}<0.05)$. TAOC levels decreased to the lowest level at the 72-h time point. QLT treatment partially resorted the TAOC levels under $\mathrm{AHH}$ (Fig. 2a).

Inflammation is an important factor for promoting acute high-altitude diseases. Hypoxia induces inflammation and the release of inflammatory factors. AHH dramatically increased the level of IL- $1 \beta$ compared with the blank condition, at the 72-h time point, although QLT suppressed the effect of $\mathrm{AHH}$ by maintaining the concentration of IL- $1 \beta$ close to the basal level (Fig. $2 b$ ). HIF- $1 \alpha$ is highly sensitive to oxygen levels and defines "the switch of hypoxia-related gene expression". In this model, AHH stimulated HIF-1 $\alpha$ expression, which was partially reversed by QLT treatment. In HAPE, high VEGF expression contributes increase pulmonary vascular permeability. AHH caused an increased expression of VEGF, but QLT treatment significantly inhibited VEGF levels in $\mathrm{AHH}$ lung tissues. Cytoglobin (CGB) is a hypoxic protective factor with oxygen-carrying capacity and protection against oxidative stress; erythropoietin (EPO) exerts antioxidative stress effects and steroid hormone-like antiinflammatory effects. Expression of both CGB and EPO were stimulated under $\mathrm{AHH}$ as a response to low $\mathrm{PO}_{2}$ conditions, and QLT further induced their levels to alleviate damage to the lung (Fig. 2c-e).

\section{RNAseq analysis of the differential expressed genes}

Volcano charts were plotted based on two factors, foldchange and the p-values obtained by t-test, show the significant differences in data between the two groups of samples (Fig. 3). The results showed that there were 1085 up-regulated genes compared with the blank compared to the $\mathrm{AHH}$ conditions, and 1705 down-regulated genes, predicted to be involved in the occurrence of acute high-altitude diseases. The comparison of QLTand saline-treated $\mathrm{AHH}$ rat showed that QLT induced the expression of 2219 genes, and down-regulated the

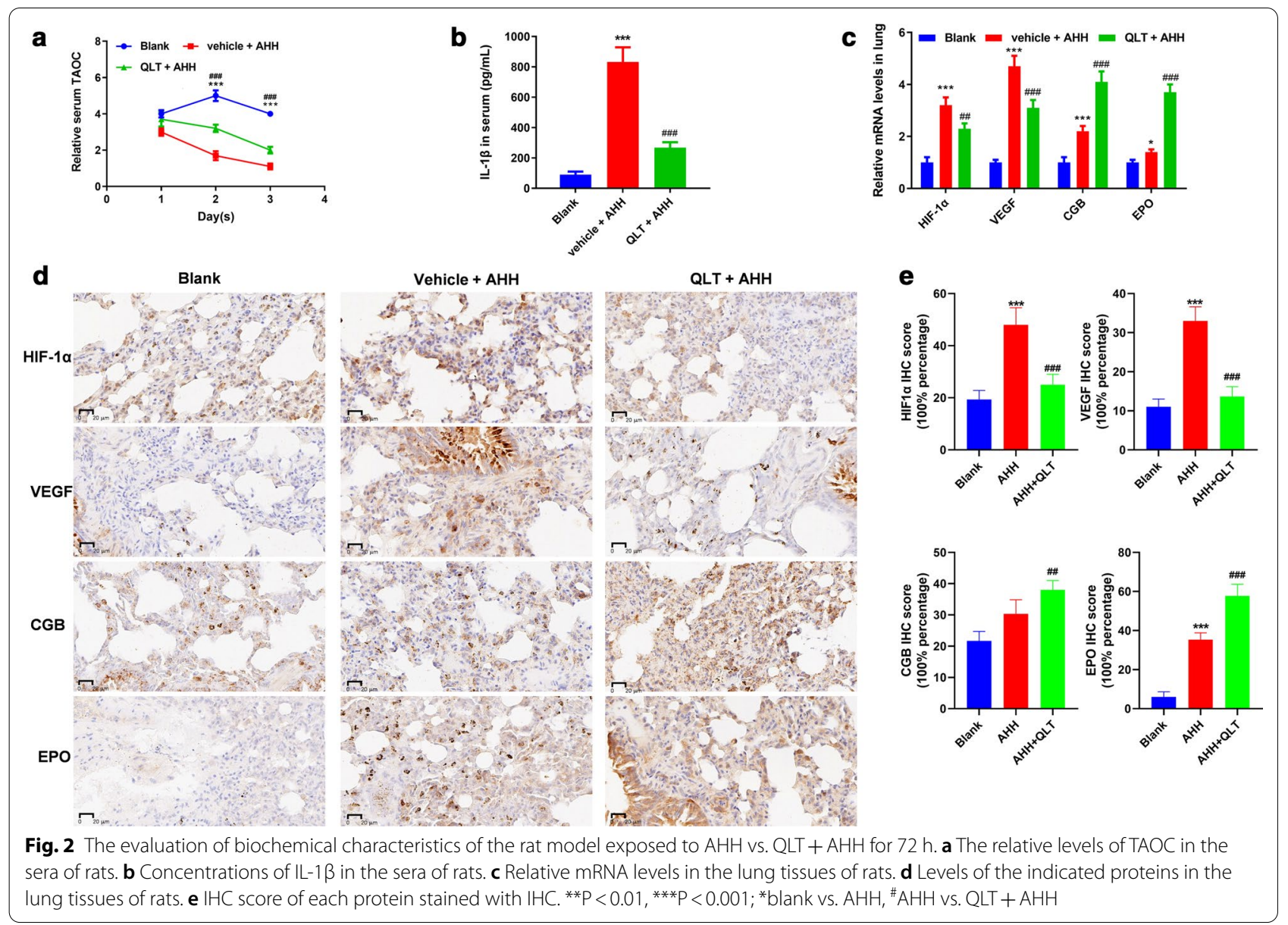



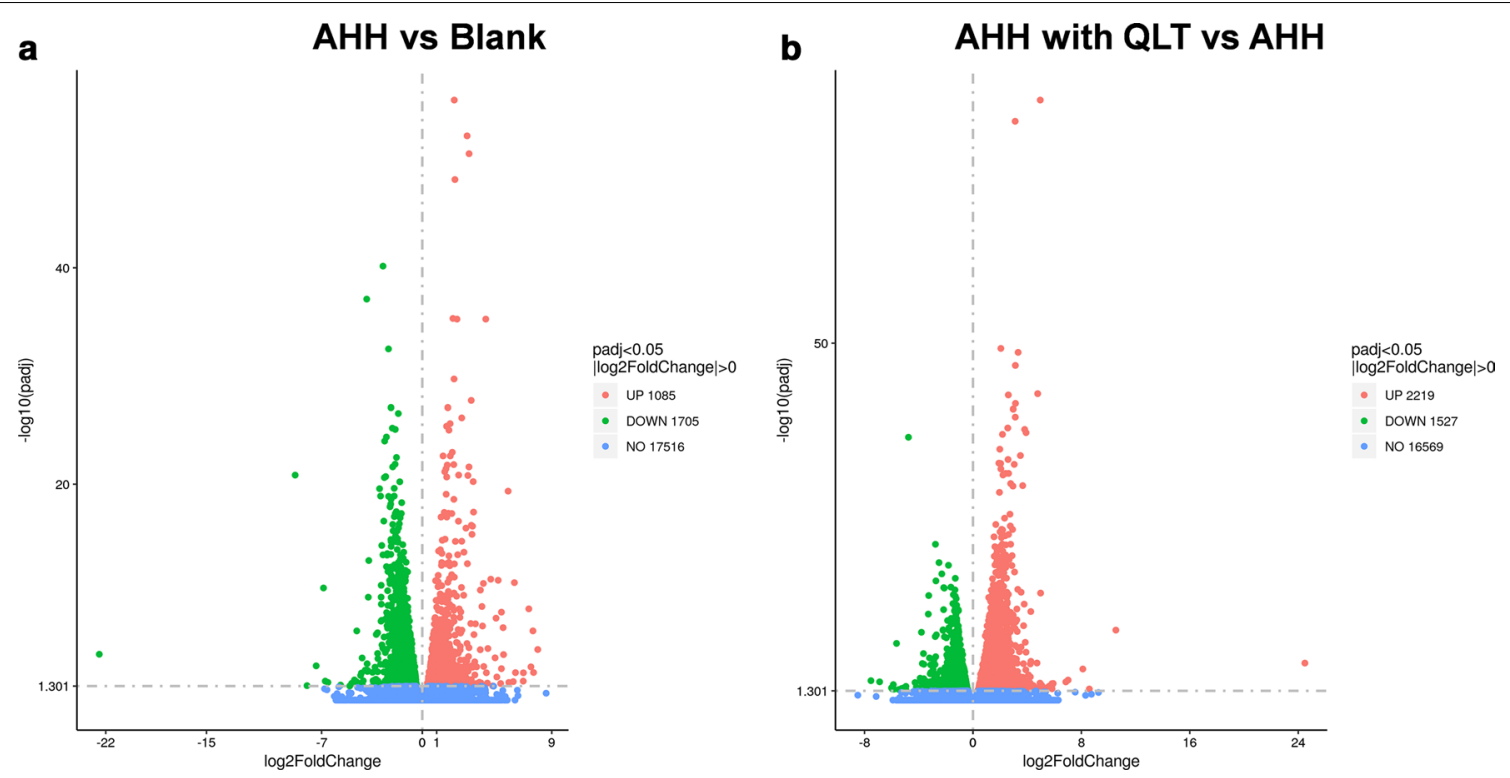

Fig. 3 Volcano plots showed differentially expressed genes in the lung tissues of the rats after exposure to AHH for $72 \mathrm{~h}$

expression of 1527 genes, which may mediate the actions of QLT in vivo.

We used hierarchical clustering to compare differentially expressed genes of representative cases as shown in the heat map (Fig. 4). The chart indicated that the threshold fold-change set in the current study can effectively separate the blank, AHH, and QLT-treated AHH rats, and the data of each case from the three groups were reproducible.

GO and KEGG analysis of the differential signaling pathway The differentially expressed genes were annotated according to the biological processes, cellular components, and molecular functions involved by BLAST2TO (Fig. 5). The major biological processes of the differentially expressed genes in the comparison between the blank and $\mathrm{AHH}$ rats were protein phosphorylation, regulation of response to stimuli, and regulation of signal transduction. The cellular component analysis showed that most were located in the nucleus, cytoskeleton, and mitochondria. The differentially expressed genes mainly play a role in protein kinase activity, GTPase binding, and ubiquitin-protein transferase activity. In the comparison of QLT- and saline-treated AHH rats, QLT activities may include the biological processes of intracellular signal transduction, protein phosphorylation, and small GTPase mediated signal transduction. The differentially expressed genes following QLT treatment were mainly associated with the endoplasmic reticulum, mitochondrial respiratory chain, and proteasome core complex. These genes responsive to QLT treatment under AHH, involved the molecular

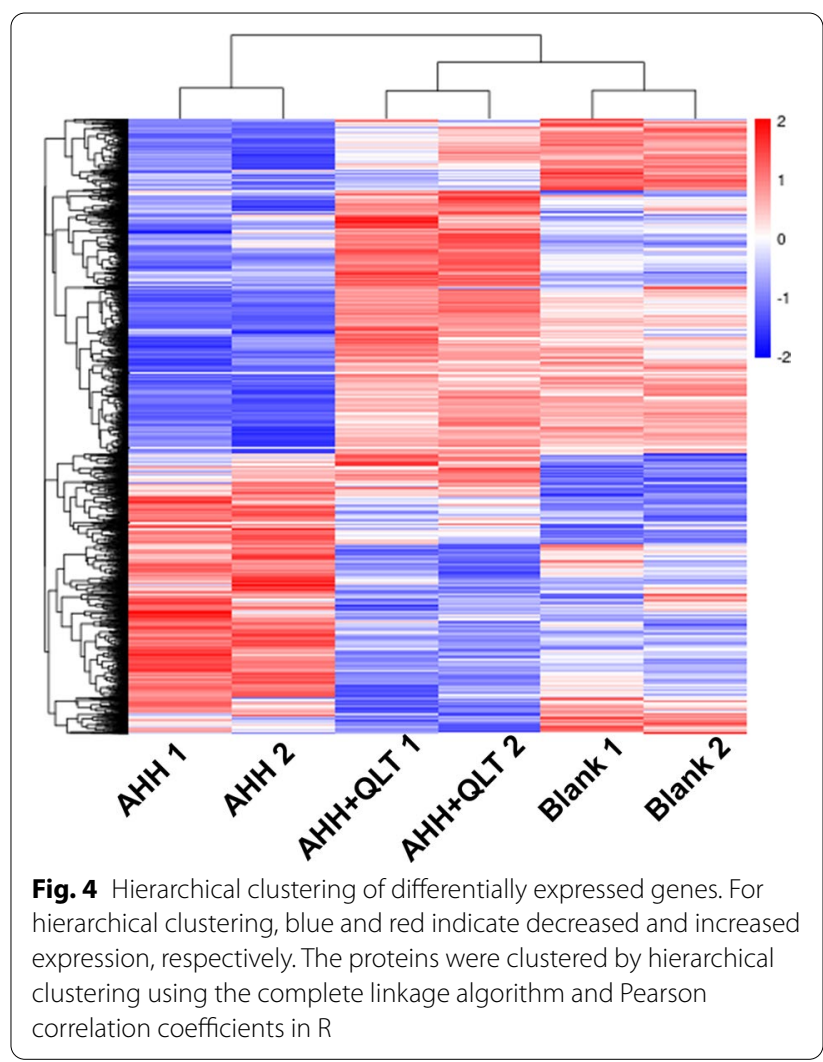

functions of protein kinase activity, GTPase binding, and protein serine/threonine kinase activity.

Pathway annotation by KEGG analysis demonstrated that the occurrence of acute high-altitude diseases was 


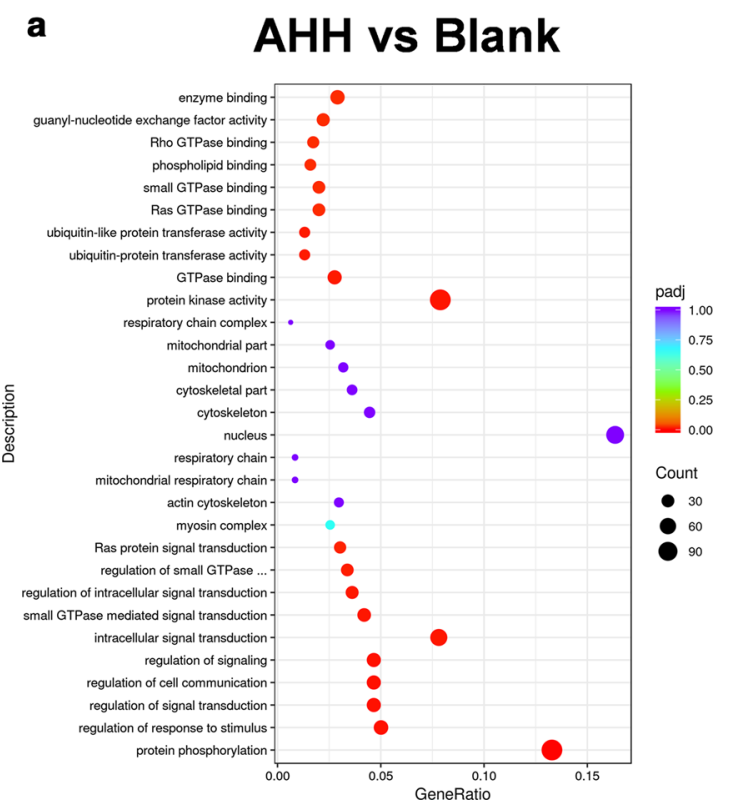

\section{b AHH with QLT vs AHH}

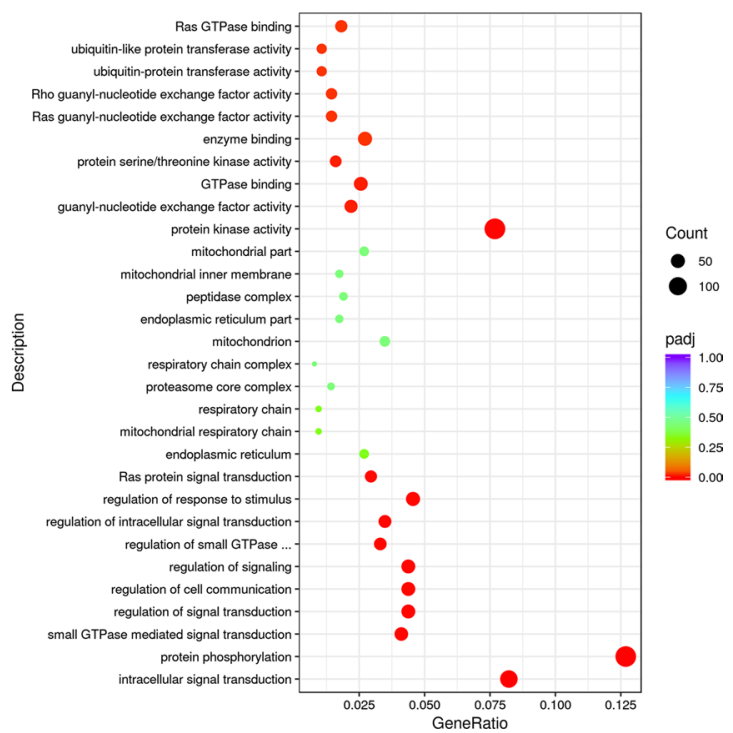

Fig. 5 GO classification of differentially expressed proteins by biological process, cellular component, and molecular function

associated with the regulation of the actin cytoskeleton, MAPK signaling pathway, and focal adhesion. Moreover, QLT may function in AHH rats by modulating focal adhesion, the AGE-RAGE signaling pathway, and oxidative phosphorylation (Fig. 6). From the bioinformatics analyses, we can begin to postulate the mechanisms involved in $\mathrm{AHH}$-induced pulmonary damage and the protective effects induced by QLT at the molecular level.

Validation of the RNAseq results and mechanism of QLT alleviate inflammatory response

By reviewing the list of the top 200 differentially expressed genes, we found that many inflammation-related genes

\section{a}

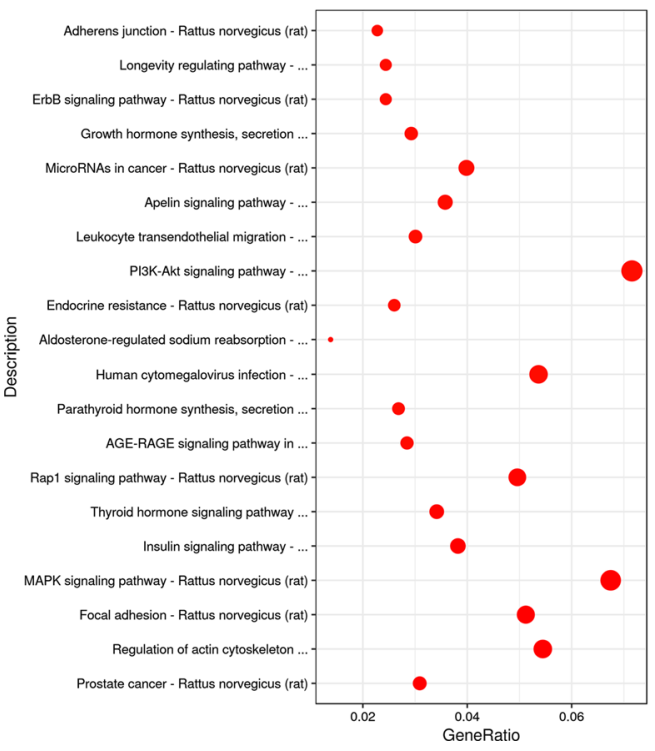

AHH vs Blank

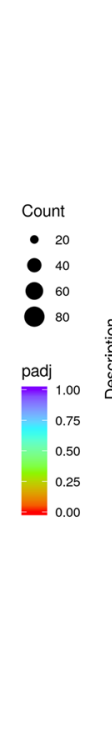

\section{b AHH with QLT vs AHH}

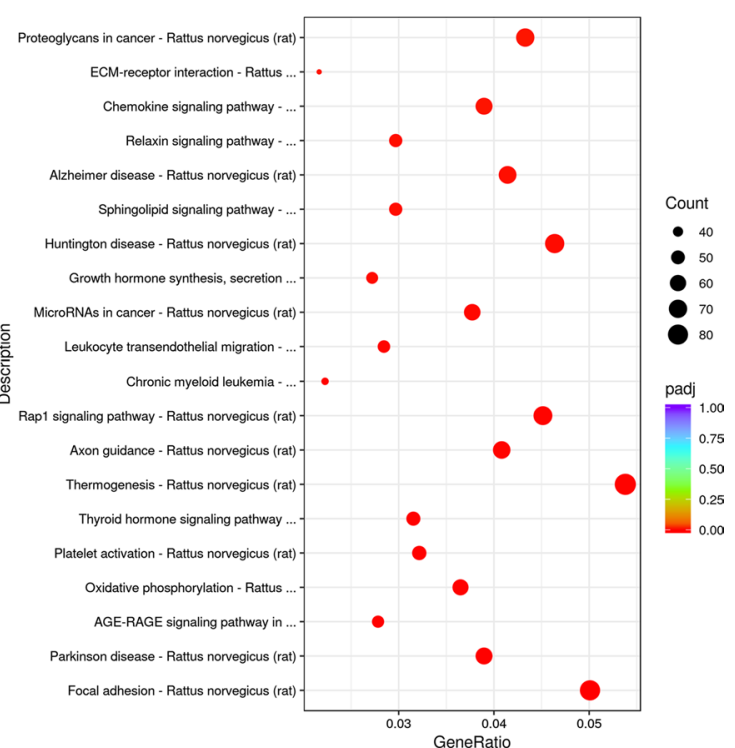

Fig. 6 KEGG analysis of the differentially expressed proteins. To the left of each plot: KEGG terms. Under each plot: the percentage of the sequence 
such as MMP8, MMP9, IL-17 $\beta$, and Timp1 were significantly upregulated in the rats exposed to $\mathrm{AHH}$, whose expression was repressed by QLT administration. Thus, we deduced that QLT might function via suppressing the inflammation brought by hypobaric hypoxia. Next, we verified the changes in mRNA and protein levels in lung tissues. As shown in Fig. 7, the trend in expression of these molecules was consistent with the sequencing data, though the differences in expression of Ccl7 and S100a8 were not significant at the mRNA level. Herein, we may conclude that QLT alleviated the inflammatory responses under AHH conditions in the rat model, which could be responsible for relieving symptoms of acute high-altitude diseases.

\section{Discussion}

TCM has shown to be effective and safe for the treatment of acute high-altitude diseases. QLT, a formula for "BuQi", "HuoXue", "TongLuo", and "LiShui" according to the theory of TCM, is composed of 3 selected medicinal materials prepared following the traditional criteria. However, as a TCM formula, QLT presents difficulties in the standardization of its composition and in clarifying the mechanisms of action involving the multi-component composition [20]. In the present study, we investigated the molecular mechanisms involved in QLT effects on alleviating the symptoms of acute high-altitude diseases. Using a rat model treated under $\mathrm{AHH}$, we evaluated the structural alterations in lung tissues using $\mathrm{HE}$ staining. Other biochemical indexes like TAOC, IL-1 $\beta$, and HIF- $1 \alpha$ also indicated that the animal models were injured following the simulated hypobaric hypoxia environment. The mRNA-Seq analyses revealed that multiple pathways were involved in the damage induced by $\mathrm{AHH}$ and the protective mechanisms activated by QLT treatment. KEGG enrichment indicated that the differentially expressed genes mainly belonged to the PI3K-AKT, focal adhesion, MAPK, and Rap1 signaling pathways. By reviewing the list of altered genes, we found many inflammatory molecules ranked high according to the fold-changes induced under AHH conditions. Moreover, genes were also sensitive to QLT treatment by showing a significantly reversed expression. Therefore, it is reasonable to deduce that QLT relieves the symptoms of acute high-altitude diseases by inhibiting the inflammatory responses induced by $\mathrm{AHH}$.

We found a large proportion of differentially expressed genes derived from phosphorylation-related pathways, which were closely associated with hypoxia stress. Previous studies [21] have confirmed that hypoxia can stimulate the PI3K/AKT pathway by phosphorylating AKT, which induces the transcription of HIF- $1 \alpha$ and stabilizes HIF- $1 \alpha$ protein, which subsequently increases the expression of EPO and VEGF to promote erythropoiesis and angiogenesis. Hypoxia results in a significant increase in reactive oxygen/nitrogen species, which act as signaling molecules for activating the MAPK pathway. Downstream JNK, ERKs, and p38 were sequentially activated by phosphorylation in response to hypoxia. Next, the MAPK pathway up-regulated the downstream targets, phosphorylated c-Jun and JunB, causing inhomogeneous vasoconstriction leading to vascular leakage and inflammation over longer exposures to hypobaric hypoxia [22]. Therefore, the findings from this study also indicated that we should pay greater attention to protein phosphorylation in rat models in the future.

In addition, we also verified the responses of inflammatory-associated proteins to QLT treatment, and they were among the most significantly differentially expressed molecules. These molecules may be effectors of QLT in this model active in relieving the symptoms of acute altitude sickness. HIF- $1 \alpha$ induces iNOS expression, which increased levels of nitric oxide and activated MMPs in various cells and tissues [23], thereby stimulating the activity of MMP9. This protease promotes the degradation of extracellular components and vascular basement membrane. Hypoxia-induced cell invasion was significantly inhibited by specifically silencing the HIF- $1 \alpha /$ MMP9 signaling pathway, indicating MMP9 plays a vital role [24]. Airway remodeling is caused by the dysregulation of MMPs and as a key regulator of the extracellular matrix, MMP9 participates in the degradation of various structural proteins including collagen and elastin. MMP9 activity in turn is affected by TIMP1, which acts as both an inhibitor and a substrate for MMP9 [25]. Our study shows that TIMP1 and MMP9 were both involved in the pathogenesis of high-altitude diseases. Ccl2, MMP9, S100a9, and S100a8 are downstream target genes of IL-17, which are involved in the pathogenesis of autoimmune responses, neutrophil recruitment, and immunity to extracellular pathogens [26]. Hypoxic conditions may enhance the permeability of pulmonary blood vessels, which in turn promote alveolar macrophages to release $\mathrm{Ccl} 2$ to recruit inflammatory monocytes, and at the same time upregulate S100a8 and S100a9 [27].

\section{Conclusion}

Herein, we analyzed the potential molecular mechanisms underlying acute high-altitude diseases such as AMS, which share similarities with other pulmonary disorders. We identified the inflammation-related effectors activated by QLT treatment against AHH, and described several phosphorylation signaling pathways involved in the pathogenesis of the disease. Although we believe that additional studies are needed to further clarify the underlying mechanisms, especially those involving protein 

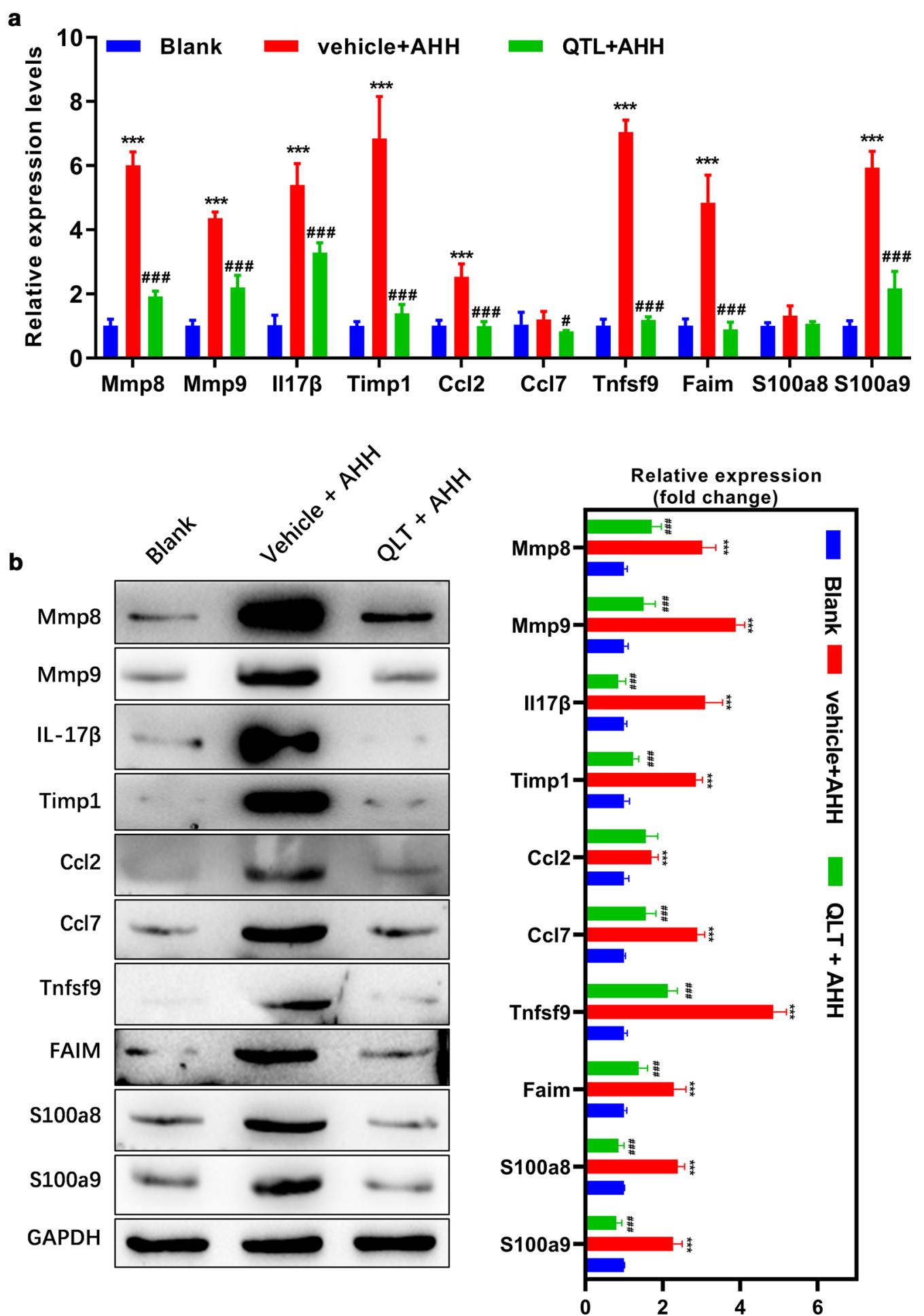

Fig. 7 Relative expressions of the inflammatory markers at the mRNA (a) and protein (b) levels. ${ }^{* *} \mathrm{P}<0.01,{ }^{* * *} \mathrm{P}<0.001 ;{ }^{*}$ blank vs. AHH, ${ }^{\#} \mathrm{AHH}$ vs. $\mathrm{QLT}+\mathrm{AHH}$ 
phosphorylation, the present study provides insights relative to the major signaling pathways of $\mathrm{AHH}$ and the protective effects of the QLT formula.

\begin{abstract}
Abbreviations
$\mathrm{PO}_{2}$ : The pressure of oxygen; AMS: Acute mountain sickness; $\mathrm{AHH}$ : Acute hypobaric hypoxia; HAPE: High-altitude pulmonary edema; TCM: Traditional Chinese medicine; QLT: Qi-Long-Tian; HE: Hematoxylin-eosin; RT: Room temperature; DEPC: Diethyl pyrocarbonate; TAOC: Total antioxidant capacity; GO: Gene Ontology; KEGG: Kyoto Encyclopedia of Genes and Genomes; qPCR: Quantitative polymerase chain reaction; GAPDH: Glyceraldehyde-3-phosphate dehydrogenase; PVDF: Polyvinylidene fluoride; PBS: Phosphate buffer saline; TNFSF9: Tumor necrosis factor ligand superfamily member 9; FAIM: Fas apoptotic inhibitory molecule; SD: Standard deviation.
\end{abstract}

\section{Authors' contributions}

$\mathrm{XF}$ and $\mathrm{CYY}$ were responsible for the experimental work and were major contributors in data collection, data analysis, and manuscript preparation. BC, KXZ, and SYC were responsible for data interpretation and visualization. YF conceived and designed the study, and was a major contributor in critically revising the manuscript. All authors read and approved the final manuscript.

\section{Funding}

This study was supported by the National Natural Science Foundation of China (Grant No.: 81860804)

\section{Availability of data and materials}

All data generated or analyzed during this study are included in this manuscript.

\section{Ethics approval and consent to participate}

The experimental protocol was approved by the Experimental Animal Care and Ethics Committees of Kunming Municipal Hospital of Traditional Chinese Medicine (Approval No.: R-082018112). Consent for participation was not applicable to this study because it does not involve the use of any human data.

\section{Consent for publication}

Not applicable.

\section{Competing interests}

The authors declare that they have no competing interests.

\begin{abstract}
Author details
${ }^{1}$ Beijing University of Chinese Medicine, Beijing 100029, China. ${ }^{2}$ Division of Lung Disease, The Third Affiliated Hospital of Yunnan University of Chinese Medicine, Kunming Municipal Hospital of Traditional Chinese Medicine, Kunming 650500, Yunnan, China. ${ }^{3}$ Function Teaching and Research Section, School of Medicine, Kunming University, Kunming 650214, Yunnan, China. ${ }^{4}$ Yunnan University of Chinese Medicine, Kunming 650500, Yunnan, China.

${ }^{5}$ The Third Affiliated Hospital of Yunnan University of Chinese Medicine, Kunming Municipal Hospital of Traditional Chinese Medicine, No. 2628 Xiangyuan Street, Chenggong District, Kunming 650500, Yunnan, China.
\end{abstract}

Received: 25 July 2020 Accepted: 31 January 2021 Published online: 12 February 2021

\section{References}

1. Hongjun $L$. Analysis of the relationship between altitude and altitude reaction from the perspective of natural selection. Agric Sci Inf. 2016;000:87-8 (in Chinese).

2. Burtscher M, Philadelphy M, Gatterer H, Burtscher J, Faulhaber M, Nachbauer W, Likar R. Physiological responses in humans acutely exposed to high altitude $(3480 \mathrm{~m})$ : minute ventilation and oxygenation are predictive for the development of acute mountain sickness. High Alt Med Biol. 2019:20:192-7.
3. Wilson MH, Newman S, Imray CH. The cerebral effects of ascent to high altitudes. Lancet Neurol. 2009;8:175-91.

4. Southard A, Niermeyer S, Yaron M. Language used in Lake Louise Scoring System underestimates symptoms of acute mountain sickness in 4- to 11-year-old children. High Alt Med Biol. 2007:8:124-30.

5. Natah SS, Srinivasan S, Pittman Q, Zhao Z, Dunn JF. Effects of acute hypoxia and hyperthermia on the permeability of the blood-brain barrier in adult rats. J Appl Physiol. 1985;2009(107):1348-56.

6. Canepa A, Chavez R, Hurtado A, Rotta A, Velasquez T. Pulmonary circulation at sea level and at high altitudes. J Appl Physiol. 1956;9:328-36.

7. Johnson NJ, Luks AM. High-altitude medicine. Med Clin N Am. 2016;100:357-69

8. Vock P, Brutsche MH, Nanzer A, Bartsch P. Variable radiomorphologic data of high altitude pulmonary edema. Features from 60 patients. Chest. 1991;100:1306-11.

9. Sophocles AM Jr. High-altitude pulmonary edema in Vail, Colorado, 1975-1982. West J Med. 1986;144:569-73.

10. Hultgren HN, Honigman B, Theis K, Nicholas D. High-altitude pulmonary edema at a ski resort. West J Med. 1996;164:222-7.

11. Maloney JP, Broeckel U. Epidemiology, risk factors, and genetics of high-altitude-related pulmonary disease. Clin Chest Med. 2005;26:395404, v.

12. Sartori C, Allemann Y, Scherrer U. Pathogenesis of pulmonary edema: learning from high-altitude pulmonary edema. Respir Physiol Neurobiol. 2007;159:338-49.

13. Stream JO, Grissom CK. Update on high-altitude pulmonary edema: pathogenesis, prevention, and treatment. Wilderness Environ Med. 2008;19:293-303.

14. Y F. Advantages of combined Traditional Chinese and Western medicine in the prevention and treatment of chronic cor pulmonale. Yunnan J Tradit Chin Med. 2007:28:46-47 (in Chinese).

15. Battistelli M, De Sanctis R, De Bellis R, Cucchiarini L, Dacha M, Gobbi P. Rhodiola rosea as antioxidant in red blood cells: ultrastructural and hemolytic behaviour. Eur J Histochem. 2005;49:243-54.

16. Qian EW, Ge DT, Kong SK. Salidroside promotes erythropoiesis and protects erythroblasts against oxidative stress by up-regulating glutathione peroxidase and thioredoxin. J Ethnopharmacol. 2011:133:308-14.

17. Pooja B. AS, Khanum F: Anti-inflammatory activity of Rhodiola rosea"a second-generation adaptogen". Phytother Res. 2009;23:1099-102.

18. Schriner SE, Avanesian A, Liu Y, Luesch H, Jafari M. Protection of human cultured cells against oxidative stress by Rhodiola rosea without activation of antioxidant defenses. Free Radic Biol Med. 2009;47:577-84.

19. Campos A, Pereira R, Vaz A, Caetano T, Malta M, Oliveira J, Carvalho FP, Mendo S, Lourenco J. Metals and low dose IR: molecular effects of combined exposures using HepG2 cells as a biological model. J Hazard Mater. 2020;396:122634.

20. Li J, Ma J, Tian Y, Zhao P, Liu X, Dong H, Zheng W, Feng S, Zhang L, Wu $M$, et al. Effective-component compatibility of Bufei Yishen formula II inhibits mucus hypersecretion of chronic obstructive pulmonary disease rats by regulating EGFR/PI3K/mTOR signaling. J Ethnopharmacol. 2020;257:112796.

21. Huang X, He Z, Jiang X, Hou M, Tang Z, Zhen X, Liang Y, Ma J. Folic acid represses hypoxia-induced inflammation in THP-1 cells through inhibition of the PI3K/Akt/HIF-1alpha pathway. PLoS ONE. 2016;11:e0151553.

22. Singh M, Yadav S, Kumar M, Saxena S, Saraswat D, Bansal A, Singh SB. The MAPK-activator protein-1 signaling regulates changes in lung tissue of rat exposed to hypobaric hypoxia. J Cell Physiol. 2018;233:6851-65.

23. McCarthy SM, Bove PF, Matthews DE, Akaike T, van der Vliet A. Nitric oxide regulation of MMP-9 activation and its relationship to modifications of the cysteine switch. Biochemistry. 2008;47:5832-40.

24. Li YY, Zheng YL. Hypoxia promotes invasion of retinoblastoma cells in vitro by upregulating HIF-1alpha/MMP9 signaling pathway. Eur Rev Med Pharmacol Sci. 2017;21:5361-9.

25. McGillick EV, Orgeig S, Morrison JL. Structural and molecular regulation of lung maturation by intratracheal vascular endothelial growth factor administration in the normally grown and placentally restricted fetus. J Physiol. 2016;594:1399-420. 
26. Silva-Santos B. Driving $\mathrm{IL}-17(+)$ gammadelta T-cell migration in allergic reactions: a new "inflammatory" role for the "homeostatic" chemokine CCL25. Eur J Immunol. 2012;42:1097-101.

27. Chao J, Viets Z, Donham P, Wood JG, Gonzalez NC. Dexamethasone blocks the systemic inflammation of alveolar hypoxia at several sites in the inflammatory cascade. Am J Physiol Heart Circ Physiol. 2012;303:H168-177.

\section{Publisher's Note}

Springer Nature remains neutral with regard to jurisdictional claims in published maps and institutional affiliations.
Ready to submit your research? Choose BMC and benefit from:

- fast, convenient online submission

- thorough peer review by experienced researchers in your field

- rapid publication on acceptance

- support for research data, including large and complex data types

- gold Open Access which fosters wider collaboration and increased citations

- maximum visibility for your research: over 100M website views per year

At BMC, research is always in progress.

Learn more biomedcentral.com/submissions 